\title{
Estudo das agressões por cães, segundo tipo de interação entre cão e vítima, e das circunstâncias motivadoras dos acidentes, município de São Paulo, 2008 a 2009
}

\author{
[Study of aggression by dogs, according to the kind of interaction between dog and \\ victim and motivating circumstances in the accidents, Municipality \\ of São Paulo, 2008 to 2009]
}

\author{
N.T. Paranhos, E.A. Silva, F. Bernardi, M.C.N.C. Mendes, D.M.A.G. Junqueira, I.O.M. Souza, \\ J.O.M. Albuquerque, J.C.M. Alves, M.N.P. Machado \\ Coordenação de Vigilância em Saúde (Covisa) - Secretaria Municipal de Saúde (SMS) - Prefeitura \\ Municipal de São Paulo (PMSP) - Santana, São Paulo, SP
}

\begin{abstract}
RESUMO
Caracterizaram-se as agressões por cães, o perfil das vítimas e dos agressores e as circunstâncias desses acidentes. Calculou-se a amostra aleatória a partir de 20 mil notificações/ano de agressões por cães e realizaram-se 594 entrevistas telefônicas. A maior frequência de agressões ocorreu no sexo masculino, faixa etária de cinco a 14 anos. Lesões leves representaram $80,4 \%$. As profundas $(19,1 \%)$ ocorreram por cães de porte médio seguidas pelos de grande porte. A maioria dos cães era macho adulto, porte médio. Dos entrevistados, 52,5\% desconheciam a condição reprodutiva; 41,0\% dos cães não eram esterilizados e $6,4 \%$ eram esterilizados. Cães sem raça definida (SRD) provocaram 48,4\% dos acidentes, e 57,6\% das agressões ocorreram em locais privados. Em 56,2\% das agressões, as vítimas eram moradoras do imóvel ou pessoas conhecidas. Com animais não conhecidos a agressão mais frequente ocorreu na rua, 30,8\%, e $67,5 \%$ dos donos permaneceram com os animais. As agressões foram resultantes da interação homem-cão e devem ser estudadas para se identificarem os possíveis comportamentos que desencadeiam a agressão pelo animal.
\end{abstract}

Palavras-chave: cão, agressão canina, vigilância, causas, interação homem-cão, saúde pública

\begin{abstract}
This study aimed to characterize aggression by dogs, the profile of victims, and perpetrators and circumstances of these accidents. Understanding the interaction between victims and aggressive dogs contributes to preventing aggression. The random sample from 20.000 reports / year of attacks by dogs was calculated and 594 telephone interviews were conducted. The highest frequency of assaults occurred in males from 5 to 14 years of age. Non-severe injuries represented 80.4\%. Deep lesions (19.1\%) were caused by medium and large dogs. Most dogs were medium size adult males. Of the respondents, $52.5 \%$ were unaware of the reproductive condition, $41.0 \%$ of dogs were not sterilized and $6.4 \%$ were sterilized. Dogs without defined race caused $48.4 \%$ of accidents and $57.6 \%$ of assaults occurred in private places. In $56.2 \%$ assault victims were residents of the property or known people. Aggressions from unknown animals were more frequent on the street (30.8\%). 67.5\% remained with the animals' owners. The attacks were the result of interaction between man and dog and should be studied to identify which human and canine behaviors can trigger accidents, to understand how the interaction occurs in different situations whithin the same household, with acquaintances, on the street, and with unknown animals. Educational approaches detailing risks and prevention methods are essential.
\end{abstract}

Keywords: dog, aggression, surveillance, causes, human/dog interaction, public health

Recebido em 7 de novembro de 2011

Aceito em 26 de novembro de 2012

E-mail: noemiap@prefeitura.sp.gov.br 


\section{INTRODUÇÃO}

O município de São Paulo tem uma população canina domiciliada estimada em 2.507.401 animais e uma população humana de 10.882.121 habitantes, o que equivale a 4,34 pessoas para cada cão (Canatto, 2010). Tem-se observado uma mudança de hábitos das pessoas no contato com cães, os quais, em muitas situações, ascendem ao status de membro da família. Observa-se uma modulação artificial pelo homem da capacidade de suporte da população canina, o qual fornece as condições necessárias de abrigo, água e alimento para manutenção de espécies domésticas, tanto no domicílio, quanto no seu entorno, na condição de animais comunitários (Canatto, 2010).

O Serviço de Vigilância de Agressões foi instituído no Centro de Controle de Zoonoses em 2006, a partir da observação de animais agressores do Programa de Vigilância da Raiva. As notificações de atendimento antirrábico são as que apresentam a maior frequência entre os 49 agravos cobertos pelo Sistema de Informação de Agravos de Notificação (SINAN): $24,13 \%$ do total, seguidas das notificações por acidente de trabalho, $18,3 \%$, e de casos de dengue, $14,01 \%$.

O atendimento antirrábico inclui mordeduras, arranhaduras e lambeduras por várias espécies de mamíferos e, no período de 2007 a 2010, teve frequência anual média de 16.830 mordeduras provocadas por cães, do total de 21.317 notificações, em média, no período, o que corresponde a $79,03 \%$ do total de atendimentos antirrábicos. A definição de mordedura se refere a qualquer lesão na pele causada pelo dente de um animal (Mathews e Lattal, 1994), independentemente da intenção do ataque, sendo possível reduzir esse problema de saúde pública quando adultos e crianças aprendem a diminuir suas chances de serem mordidos (CDC, 2010).

Nas agressões por cães, há o risco de transmissão de enfermidades infecciosas, como raiva, pasteurelose, tétano, outras infecções secundárias, sequelas psicológicas e incapacidades, e pode ocorrer, ainda que mais raramente, o óbito da vítima. Devem-se também considerar os custos derivados dos tratamentos médicos e psicológicos, a abstenção ao trabalho, a observação e o controle dos animais.
Há consenso de que os cães sinalizam quando vão atacar: rosnam e mordem em inúmeras situações que podem ser motivadoras de ataques e ferimentos. Existem diferentes classificações, caracterizando a vítima ou a motivação do animal, como por dominância, medo, dor, brincadeiras; idiopática, intrassexual, aprendida, maternal, médica, protetora, predatória, redirigida (Houpt, 2006; Duffy et al., 2008).

Especialistas concordam que qualquer cão é capaz de morder, incluindo os sem história de agressão e aqueles cujo comportamento agressivo foi tratado, independentemente de receber treinamento de obediência ou ter sido socializado com crianças. Muitos acreditam que mordidas sejam resultado de provocação ou ferimento ao animal. No entanto, mesmo interações afetivas ou neutras de crianças, em que ocorrem alterações posturais, contato visual e carícias, podem ser provocativas para alguns cães (Reisner e Shofer, 2008).

A maioria dos etólogos aceita que a agressão canina e de outras espécies envolve sempre um contexto e que um cão que responde agressivamente em uma determinada situação não necessariamente o fará em outra (Serpell e Jagoe, 1995). Ainda segundo Serpell e Jagoe (1995), a agressão pode ser classificada como por dominância quando se manifesta uma tendência de alguns cães reagirem de forma agressiva a possíveis desafios da sua posição social hierárquica. Nessas circunstâncias, incluem-se situações em que o proprietário é aparentemente visto, por exemplo, como um competidor de recursos alimentares, de espaço, do local de dormir, ou em resposta a um gesto do proprietário de suposta dominância, como segurar, acariciar, limpar, restringir, punir, gritar, olhar ou mesmo inclinar-se sobre o animal. $\mathrm{O}$ conceito de dominância baseia-se na noção de que os animais competem agressivamente mais por posição social do que para obtenção de alimento ou escolha de parceiros, e tem sido utilizado para explicar a organização e a manutenção da estrutura social de diferentes grupos de animais.

Serpell e Jagoe (1995) definiram agressão do tipo territorial como uma resposta em que um cão apresenta a tendência a reagir agressivamente quando um intruso entra em seu território ou nas vizinhanças deste, e se manifesta 
contra pessoas ou animais desconhecidos que se aproximam do seu espaço. A definição desse espaço muitas vezes é maior do que aquele que se entende como o do cão e pode ser o canil, o quintal, a casa ou o quarteirão (CCZ, 2004).

As agressões podem ocorrer nos contextos de expressões de dominância, defesa territorial, competição por alimento, proteção de membros jovens da matilha ou de outros indivíduos. Podem acontecer por dor, medo e também podem envolver componentes de comportamento predatório interespecífico. Estudos demonstram que a mordedura é componente do comportamento predatório em canídeos, embora os animais mais sociais mostrem baixos níveis de agressão interespécie (Lockwood, 1998).

O presente trabalho objetiva caracterizar as agressões por cães, o perfil das vítimas de agressão e dos agressores e as circunstâncias em que esses acidentes aconteceram.

\section{MATERIAL E MÉTODOS}

Foi realizado um inquérito populacional com amostra aleatória simples a partir dos registros de atendimento antirrábico no município de São Paulo. Foram amostradas as notificações de atendimento antirrábico de agressões por cães, por meio do Sistema de Informação de Agravos de Notificação (SINAN), em São Paulo, tendo em conta a média de 20 mil notificações/ano. A média de casos notificados por mês em 2007 foi de 1.296. Utilizou-se o software Epi Info® para o cálculo do tamanho da amostra, considerando-se que $95 \%$ das estimativas poderiam diferir do valor populacional por, no máximo, 5\%; o resultado foi de 363. Estimandose não resposta de $35 \%$, o tamanho da amostra a ser sorteado seria de 559 casos.

Com a média mensal de 1.200 notificações e uma amostra sistemática, sorteou-se uma ficha a cada dez, segundo a ordem de incorporação do registro no banco de dados.

A estratégia escolhida foi a de entrevista telefônica e selecionaram-se para sorteio apenas as fichas cujo campo "número do telefone" estivesse completo.

As entrevistas foram realizadas por telefone, por técnicos do Centro de Controle de Zoonoses da
Covisa, mediante um questionário padronizado e previamente testado.

Para a apresentação dos resultados, foram calculados intervalos de confiança de $95 \%$ das frequências e realizados testes de comparação entre duas proporções (estatística z), utilizando-se os pacotes estatísticos EpiInfo® e Minitab®, respectivamente. Considerou-se diferença estatisticamente significante $\mathrm{P}<0,05$.

Para classificar o motivo da agressão, foi utilizada uma adaptação da categorização criada por Palacio et al. (2005)

\section{RESULTADOS E DISCUSSÃO}

Foram realizadas 594 entrevistas, entre 09/2008 e 09/2009, e as agressões ocorreram entre 01/2008 e 08/2009. No total da amostra, a maior frequência de agressões ocorreu em pessoas do sexo masculino, 53,4\% $(\mathrm{P}<0,05)$. A faixa etária mais agredida foi de cinco a 14 anos, 28,1\% ( $\mathrm{P}<0,0001)$, e nessa faixa, mais frequentemente, as agressões foram resultantes de brincadeiras.

O estudo de Palacio et al. (2005) ressaltou que crianças menores, por curiosidade e inexperiência, expõem-se mais, ficam mais tempo nas ruas e são menos supervisionados por pessoas adultas, tendo maior dificuldade para se defender. Segundo Gershman (1994), 51\% das vítimas tinham 12 anos ou menos.

Reisner (2008) encontrou resultados sugestivos de que os proprietários frequentemente têm conhecimento limitado do comportamento canino e não sabem dos fatores que aumentam os riscos de agressões em crianças. Brincadeiras bruscas, correrias, barulho, invasão do que o animal considera seu "território" podem despertar agressividade, e as crianças, em geral, não sabem reconhecer expressões corporais e faciais, grunhidos, retração dos lábios, olhar penetrante, orelhas e cauda eretas, entre sinais com os quais o animal comunica que vai atacar (Mathews e Lattal, 1994).

Schalamon (2006) observou a incidência anual de mordidas por cães de 0,5 por 1.000 crianças entre zero e 16 anos de idade. No presente estudo, foi de 0,077 por 1.000 crianças entre zero e 14 anos, no entanto, no caso do sexo 
masculino, aumentou para 0,104 por 1.000 nesta faixa.

$\mathrm{Na}$ avaliação do que motivou a agressão, pode-se considerar também o fato de existir ou não familiaridade do cão com o ser humano. Os cuidadores diretos são considerados os mais familiares. A circunstância de agressão pode ser um fator de diferenciação num acidente de agressão (Houpt, 2006).

Para se verificar a existência de possíveis diferenças de interação homem-animal, segundo o grau de contato do agredido e do agressor, estabeleceu-se a variável "proximidade", composta por informações coletadas sobre o grau de contato, a condição de o animal ser conhecido previamente, a frequência, o tempo de interação e o local de convívio - própria casa, vizinho, rua, etc. -, e verificou-se que $44,1 \%(40,1-48,2 \%)$ eram desconhecidos, $32,4 \%$ (28,7 - 36,4\%) bem conhecidos e $22,6 \%(19,4-26,3 \%)$ pouco conhecidos.

A maior frequência de lesões nas pessoas agredidas ocorreu nos membros inferiores, seguidas de agressões na mão, membros superiores e cabeça. Vítimas próximas do animal tiveram lesões mais frequentemente nas mãos, $45,0 \%$ (37,8 - 52,4\%), e membros superiores, $15,7 \%(10,9-21,75 \%)$. Em pessoas com pouco contato com seus agressores, as lesões prevaleceram nos membros inferiores, 39,1\% $(30,8-47,9 \%)$, seguidas das mãos, 20,3\% (13,8 $28,1 \%)$. No caso de animais desconhecidos, as pessoas foram mais agredidas nos membros inferiores, 49,8\% (43,6 - 56,0\%), e membros superiores, $17,2 \%(12,9-22,4 \%)$.

A maioria das lesões foi leve e representou $80,4 \%$. As lesões profundas, $19,1 \%$, foram provocadas por cães de porte médio, 48,7\% (39,2 - 58,3\%), e grande, $39,8 \%$ (30,7-49,5), e as lesões profundas foram provocadas mais frequentemente pelos cães de grande porte, 32,3\%, (IC95\% 24,9 - 41,1). Não foram registrados óbitos. A maioria dessas lesões evoluiu para cura sem sequelas em 97,3\% (95,56 - 98,4) dos casos. As lesões na cabeça ocorreram em $8,5 \%$ dos casos. Nessa região do corpo, $74,5 \%(61,0$ - 85,3\%) do grupo etário de zero a 14 anos foi o mais acometido. Overall e Love (2001) verificaram que a maioria das lesões em adultos ocorreu nas extremidades, enquanto em mais de $70 \%$ dos casos em crianças, foram lesionadas a cabeça, o pescoço e a face.

Os acidentes foram provocados em $48,4 \%$ por cães sem raça definida, pela raça Poodle em $7,4 \%$ (5,5 - 9,9\%), Pitbull em 5,9\% (4,2 - 8,2\%) e Pastor Alemão em 5,7\% (4,1 - 8,0\%). Raças com maior número de indivíduos podem causar mais agressões. Invariavelmente cães de raças grandes são um problema porque o dano pode ser maior ao morder. Entretanto, cães de raças pequenas também mordem e são capazes de causar ferimentos graves (Beaver et al., 2001).

Entre várias razões pelas quais não é possível calcular uma taxa comparativa de agressão entre as raças, inclui-se o fato de que a raça do animal mordedor pode não ser precisamente registrada, e os cães sem raça definida são comumente descritos como se fossem de uma raça específica. Por outro lado, o número real de mordidas que ocorrem em uma comunidade não é conhecido, especialmente se não resultar em graves ferimentos. E, por último, o número de cães de uma raça especial ou uma combinação de raças em uma comunidade não é conhecido (Beaver e Baker et al., 2001; Wright 1991). No estudo atual, nada se pode afirmar comparativamente à distribuição na população canina, em razão da falta de disponibilidade de dados relevantes sobre raça.

A maioria dos cães agressores era adulta, 82,4\%, de porte médio, 51,7\%, e macho, 64,3\%, $(\mathrm{P}<0,0001)$. Entre os animais conhecidos, os machos agrediram 3,39 vezes mais do que as fêmeas. Patrick e Rourke (1998), em um estudo com 295 cães agressores, identificaram o sexo de $92,5 \%$ dos animais, e $65,6 \%$ desses eram machos. Beck (1975) identificou entre os agressores $70 \%$ de machos. Machos tendem a morder 6,2 vezes mais do que fêmeas, e os animais não esterilizados tendem a morder 2,6 vezes mais (Sacks et al., 2000). O cão agressor era de pessoa conhecida em $44 \%$ dos casos, vivia na mesma casa que a vítima em $28,7 \%$ das situações, era de rua ou tinha dono desconhecido em $24,3 \%$ dos casos.

Métodos de prevenção de agressão incluem controle animal, registro, legislação, obediência e socialização, tratamento e orientação com médicos veterinários especialistas em comportamento. A esterilização diminui para um 
terço a probabilidade de mordeduras (Cornwell, 1997).

Do total, 594 dos entrevistados, 52,5\%, não souberam informar sobre a condição reprodutiva do animal, 41,0\% relataram que o cão não era castrado e 6,4\% disseram que o cão era castrado. No entanto, essa informação foi variável de acordo com o grau de proximidade das pessoas envolvidas, e entre as pessoas que conheciam bem o animal, $89,0 \%$ conheciam sua condição reprodutiva. Desses animais, $14,7 \%$ eram esterilizados e $74,3 \%$ não eram esterilizados, o que significa que diminuiu para $11,0 \%$ o percentual de pessoas que não conheciam essa informação (Tab. 1). Nesse caso, os animais não esterilizados agrediram 5,07 vezes mais que os animais esterilizados e $57,6 \%$ das agressões ocorreram em locais privados, como quintais e garagens, dentro dos domicílios, através de grades ou portões, ou em estabelecimentos comerciais/industriais. Nesses locais, a motivação de agressão mais frequente foi a defesa de território, 25,4\% (21,0 -30,5\%), seguida da condição em que a pessoa brincava com o animal. Quando a agressão ocorreu em local público, em ruas, praças e parques, o cão não usava restrição em $87,4 \%$ dos casos. Nessa situação, a maior frequência das agressões ocorreu quando a vítima passava pelo local, $35,3 \%(29,4-41,6)$.

Tabela 1. Condição reprodutiva e grau de proximidade entre vítima e animal agressor no município de São Paulo, 2009

\begin{tabular}{cccccc}
\hline Condição reprodutiva Proximidade & Não sabe & $\begin{array}{c}\text { Não } \\
\text { esterilizado }\end{array}$ & Esterilizado & Total \\
\hline Animal bem & $\mathrm{n}$ & 21 & 142 & 28 & 191 \\
conhecido & $\%$ & $11,0 \%$ & $74,3 \%$ & $14,7 \%$ & $100,0 \%$ \\
& IC95\% & $6,9-16,3$ & $67,5-80,4$ & $10,0-20,5$ & - \\
Animal pouco & $\mathrm{n}$ & 74 & 53 & 7 & 134 \\
conhecido & $\%$ & $55,2 \%$ & $39,6 \%$ & $5,2 \%$ & $100,0 \%$ \\
& IC95\% & $46,4-63,8$ & $31,2-48,4$ & $2,1-10,5$ & - \\
Animal & $\mathrm{n}$ & 212 & 45 & 2 & 259 \\
desconhecido & $\%$ & $81,9 \%$ & $17,4 \%$ & $0,8 \%$ & $100,0 \%$ \\
& IC95\% & $76,6-86,4$ & $13,0-22,5$ & $0,1-2,8$ & - \\
\hline \multirow{2}{*}{ Total } & $\mathrm{n}$ & 310 & 242 & 38 & 590 \\
& $\%$ & $52,5 \%$ & $41,0 \%$ & $6,4 \%$ & $100,0 \%$ \\
\hline
\end{tabular}

No total da amostra, a mais frequente motivação de agressões informada foi defesa de território, $15,3 \%(12,6-18,5)$, seguida por situações em que a vítima estava passando pelo local da agressão, $15,1 \%(12,4-18,3)$, a vítima brincava com o animal agressor $11,9 \%(9,5-14,9)$, ou em brigas entre animais, $6,2 \%(4,5-8,6)$.

Nos casos em que a vítima conhecia bem o cão agressor, as causas mais frequentes de agressão ocorreram quando a vítima brincava com o animal, 17,2\% (12,1-23,3), em situações de briga entre animais, $13,5 \%(9,0-19,2)$, por mexer nos pertences do cão, $8,9 \%(5,2-13,8)$, e ao cuidar dele, $8,9 \%(5,2-13,8)$.

Quando as pessoas conheciam pouco os animais, as agressões que mais predominaram foram do tipo "territorial", $28,4 \%(20,9-36,8)$, ou quando a vítima brincava com o animal, 13,4\% (8,2-20,4), ao passar pelo local de agressão, 11,9\%
(7,0-18,7), ou sem que o motivo pudesse ser identificado, $7,5 \%(3,6-13,3)$.

As agressões tiveram diferentes incidências quando se observaram os grupos etários e o sexo das vítimas (Tab. 2).

Quando as pessoas desconheciam o animal, as agressões mais frequentes foram nas situações em que a vítima passava pelo local, $28,4 \%$ (23,0-34,2), ou de defesa de território, $18,0 \%$ $(13,5-23,2)$, quando o animal fugiu da casa, $10,3 \%(6,9-14,7)$, ou quando a vítima andava de bicicleta, 8,0\% (5,0-12,0). Borchelt et al. (1983) identificaram a agressão por defesa do território em $45 \%$ de um total de 2.538 casos analisados. Crianças do sexo masculino foram mais agredidas, provavelmente por se expor mais nas brincadeiras, desencadeando o instinto predador do animal, e talvez por desconhecer os riscos. 


\section{Paranhos et al.}

Tabela 2. Distribuição e incidência (por 1.000 pessoas) das agressões, segundo os grupos etários e o sexo, no município de São Paulo, 2009

\begin{tabular}{|c|c|c|c|c|}
\hline \multirow{2}{*}{ Grupo etário } & \multirow{2}{*}{ Sexo } & \multirow{2}{*}{$\mathrm{n}$} & \multicolumn{2}{|c|}{ Incidência agressões: 1000 pessoas } \\
\hline & & & por sexo & por grupo etário \\
\hline \multirow{2}{*}{$0-14$} & $\mathrm{M}$ & 139 & 0,104078 & \multirow{2}{*}{0,0772} \\
\hline & $\mathrm{F}$ & 64 & 0,049246 & \\
\hline \multirow{2}{*}{$15-29$} & M & 45 & 0,036563 & \multirow{2}{*}{0,0399} \\
\hline & $\mathrm{F}$ & 53 & 0,043188 & \\
\hline \multirow{2}{*}{$30-44$} & M & 49 & 0,039506 & \multirow{2}{*}{0,0362} \\
\hline & $\mathrm{F}$ & 44 & 0,033190 & \\
\hline \multirow{2}{*}{$45-59$} & M & 36 & 0,039102 & \multirow{2}{*}{0,0492} \\
\hline & $\mathrm{F}$ & 63 & 0,057680 & \\
\hline \multirow{2}{*}{$60-74$} & M & 39 & 0,092933 & \multirow{2}{*}{0,0741} \\
\hline & $\mathrm{F}$ & 34 & 0,060194 & \\
\hline \multirow{2}{*}{75 ou mais } & $\mathrm{M}$ & 7 & 0,061527 & \multirow{2}{*}{0,0730} \\
\hline & $\mathrm{F}$ & 17 & 0,079128 & \\
\hline \multirow{2}{*}{ Total } & $\mathrm{M}$ & 315 & 0,060039 & \multirow{2}{*}{0,0537} \\
\hline & $\mathrm{F}$ & 275 & 0,047943 & \\
\hline
\end{tabular}

Patrick e Rourke (1998) encontraram 87\% dos cães agressores com donos desconhecidos, e do total dos ataques $45 \%$ foram provocados pela própria vítima. A maioria dos casos de agressão, tanto no total da amostra estudada como nos casos em que os animais eram conhecidos, ocorreu no endereço do cão (Tab. 3). Quando o animal era desconhecido, o local mais frequente da agressão foi a rua, nas imediações do endereço da vítima, $31,2 \%(25,6-37,2)$. Borchelt et al. (1983) observaram que os ataques ocorreram mais frequentemente ou no endereço de residência do cão, ou nas suas proximidades.

Tabela 3. Distribuição dos casos de agressão por cão, de acordo com o endereço da agressão e a proximidade com o animal, no município de São Paulo, 2009

\begin{tabular}{|c|c|c|c|c|c|c|c|c|}
\hline \multicolumn{2}{|l|}{$\begin{array}{l}\text { Endereço de agressão } \\
\text { Proximidade }\end{array}$} & $\begin{array}{l}\text { Endereço } \\
\text { do cão }\end{array}$ & $\begin{array}{c}\text { Rua/imed } \\
\text { vítima }\end{array}$ & $\begin{array}{l}\text { Rua/imed } \\
\text { cão }\end{array}$ & Outros & $\begin{array}{l}\text { Endereço } \\
\text { da vítima }\end{array}$ & $\begin{array}{l}\text { Não } \\
\text { sabe }\end{array}$ & Total \\
\hline \multirow{4}{*}{ Animal bem conhecido } & $\mathrm{n}$ & 159 & 3 & 11 & 14 & 5 & - & 192 \\
\hline & $\%$ & $82,8 \%$ & $1,6 \%$ & $5,7 \%$ & $7,3 \%$ & $2,6 \%$ & - & $100,0 \%$ \\
\hline & IC95\% & $76,7-87,9$ & $0,3-4,5$ & $2,9-10,0$ & $4,0-11,9$ & $0,9-6,0$ & - & - \\
\hline & $\mathrm{n}$ & 75 & 29 & 12 & 9 & 9 & - & 134 \\
\hline \multirow[t]{2}{*}{ Animal pouco conhecido } & $\%$ & $56,0 \%$ & $21,6 \%$ & $9,0 \%$ & $6,7 \%$ & $6,7 \%$ & - & $100,0 \%$ \\
\hline & IC95\% & $47,1-64,5$ & $15,0-29,6$ & $4,7-15,1$ & $3,1-12,4$ & $3,1-12,4$ & - & - \\
\hline \multirow{3}{*}{ Animal desconhecido } & $\mathrm{n}$ & 46 & 81 & 68 & 53 & 10 & 2 & 260 \\
\hline & $\%$ & $17,7 \%$ & $31,2 \%$ & $26,2 \%$ & $20,4 \%$ & $3,8 \%$ & $0,8 \%$ & $100,0 \%$ \\
\hline & IC95\% & $13,3-22,9$ & $25,6-37,2$ & $20,9-31,9$ & $15,7-25,8$ & $1,9-7,0$ & $0,1-2,8$ & \\
\hline \multirow{3}{*}{ Total } & $\mathrm{n}$ & 284 & 114 & 92 & 77 & 24 & 2 & 593 \\
\hline & $\%$ & $47,9 \%$ & $19,2 \%$ & $15,5 \%$ & $13,0 \%$ & $4,0 \%$ & $0,3 \%$ & $100,0 \%$ \\
\hline & IC95\% & $43,8-52,0$ & $16,2-22,7$ & $12,7-18,7$ & $10,4-16,0$ & $2,7-6,1$ & $0,1-1,4$ & - \\
\hline
\end{tabular}

As agressões ocorreram menos frequentemente aos domingos, $8,1 \%$, e entre os outros dias não houve diferença quanto à ocorrência de agressões.

Palacio et al. (2005) enfatizaram que o prognóstico de reintrodução do animal agressor no convívio depende da intensidade e da previsibilidade da agressão, do conhecimento em relação ao tempo em que tal comportamento se apresenta, da possibilidade de a família viabilizar tratamento, reeducação ou manejo adequado, do perfil da pessoa agredida e da impulsividade do animal.

No estudo atual, em $67,5 \%$ dos casos, os animais agressores permaneceram com o dono, $5,6 \%$ foram soltos nas ruas e $3,6 \%$ foram doados. Nas situações em que os animais eram bem conhecidos, o percentual de permanência com os 
donos cresceu para $84,4 \%$. No caso de animais pouco conhecidos, $78,2 \%$ também ficaram com os donos.

Nas situações onde animais com histórico de agressão, que permanecem no domicílio, ou são encaminhados para doação, ou estejam abandonados, necessitam do implemento de estratégias para reduzir a incidência de mordidas.

Borchelt et al. (1983) identificaram que, em alguns casos de agressões, os proprietários não se preocuparam em inibir o comportamento agressivo de seus animais em relação a pessoas estranhas; pelo contrário, essa atitude, em certas circunstâncias, foi estimulada.

Beck e Jones (1985) constataram que proprietários são mais mordidos não apenas pelo fato de estarem mais expostos ao contato com o cão como também porque tendem a não se esquivar de outros cães.

Várias medidas preventivas podem ser adotadas por proprietários ou futuros proprietários (CDC, 2010): consulta a profissionais especialistas em comportamento; estudo de raças e temperamentos mais adequados; convívio com o animal antes da adoção; esterilização; maior cuidado de famílias com crianças ao adotar um cão e supervisão permanente às crianças quando em contato com um cão.

\section{CONCLUSÃO}

Conclui-se que crianças de cinco a 14 anos têm maior probabilidade de serem mordidas. É imprescindível estabelecer abordagens educativas sobre risco e prevenção de agressão, considerando níveis de contato com os animais e locais de convívio, tendo em conta que a agressividade é resultante da interação homem-cão e precisa ser estudada sob essa ótica, para identificação dos comportamentos humanos e caninos que desencadeiam a agressão pelo animal.

\section{REFERÊNCIAS}

BECK, A.M.; JONES, B.A. Unreported Dog Bites in Children. Pub. Health Rep., v.100, p.315-321, 1985.

BECK, A.M.; LOORING, H.; LOCKWOOD, R. The Ecology of Dog Bite Injury in St. Louis, Missouri. Pub. Health Rep., v.90, p.262-267, 1975.

BEAVER, B.V.; BAKER, M.D. et al. A community approach to dog bite prevention. Task Force on Canine Aggression and Human-Canine Interactions. Vet Med Today: Canine Aggression Task Force. J. Am. Vet. Med. Assoc, v.218, 2001. Disponível em https://www.avma.org/public/Health/Documents/ dogbite.pdf

BORCHELT, P.L.; LOCKWOOD, R.; BECK, A.M.; VOITH, V.L. Attacks by Packs of Dogs Involving Predation on Human Beings. Pub. Health Rep., v.98, p.57-66, 1983.

CANATTO, B.D. Caracterizações das populações de cães e gatos domiciliadas no município de São Paulo. 2010. 81f. Dissertação (Mestrado em Ciências). Faculdade de Medicina Veterinária e Zootecnia da Universidade de São Paulo.

CCZ - Centro de Controle de Zoonoses/ COVISA/SMS, "Criando um amigo" Manual de Prevenção contra agressões por cães e gatos, Prefeitura do Município de São Paulo, 2004. Disponível em: <http:// www.pclq.usp.br/gcaa/manual_educador.pdf > Acessado em 03 jun 2011.

CDC - Centers for Disease Control and Prevention. 2010. Disponível em: <http://www.cdc.gov/HomeandRecreationalSafet y/Dog-bites/biteprevention.html Acessado em 06 jun 2011.

CORNWELL, J.M. Dog Bite prevention: Responsible pet ownership and animal safety. $J$. Am. Vet. Med. Assoc, v.210, p.1147-1148, 1997.

DUFFY, D.L.; HSU, Y.; SERPELL, J.A. Breed Differences in canine aggression. App. Anim. Behav. Sci., v.114, p.1-20, 2008.

GERSHMAN, K.A.; SACKS, J.J.; WRIGHT, J.C.; Which Dogs Bite? Case-Control Study off Risk factors for dogs biting. Pediatrics, v.93, p.913-917, 1994. 
HOUPT, K.A. Terminology Think Tank: Terminology of aggressive behavior. J. Vet. Behav., v.1, p.39-41, 2006.

LOCKWOOD, R. The etology and epidemiology or canine aggression. In: Serpell J.A. (Ed) The domestic Dog: Its evolution, Behaviour and Interactions with People. Cambridge: Cambridge University Press, 1998. p.131- 138.

MATHEWS, J.R; LATTAL, K.A. A behavioral analysis of dog bites to children. J. Dev. Behav. Pediatr., v.15, p.44-52, 1994.

OVERALL, K.L.; LOVE, M. Dog bites to humans - demography epidemiology, injury and risk. J. Am. Vet. Med. Assoc, v.218, p.1923-1934, 2001.

PALACIO, J.; LEÓN, M.; GARCÍA-BELENGUER, S. Aspectos epidemiológicos de las mordeduras caninas. Gac. Sanit., v.19, p.50-58, 2005.

PATRICK, G.R.; O'ROURKE, K.M. Dog And Cat Bites: Epidemiologic Analyses Suggest Different Prevention Strategies. Pub. Health Rep., v.113; p.252-257, 1998.
REISNER, I.R.; SHOFER, F.S. Effects of gender and parental statusd on knowledge and attitudes of dog owners regarding dog agression toward children. J. Am. Vet. Med. Assoc, v.233, p.14121419, 2008.

SACKS, J.J.; SINCLAIR, L.; GILCHRIST, J. et al. Breeds of dogs involved in fatal human attacks in the United States between 1979 and 1998. J. Am. Vet. Med. Assoc., v.217, p.836-840, 2000.

SCHALAMON, J.; AINOEDHOFER, H.; SINGER, G. et al. Analysis of Dog Bites in Children Who Are Younger Than 17 Years. Pediatrics, v.117, p.374-380, 2006.

SERPELL, J.; JAGOE, J.A. Ealrly experience and development of behaviour. The Domestic Dog: Its Evolution, Behaviour and Interactions with People. Cambridge: Cambridge University Press. 1995. p.82-102.

WRIGHT, J.C. Canine aggression toward people: bite scenarios and prevention. Vet. Clin. North Am. Small Anim. Pract., v.21, p.299-314, 1991. 